Case Report

\title{
Silane Heat Treatment Could Eliminate the Hydrofluoric Acid Etching of Lithium Disilicate Overlays: A Four-Year Follow-Up
}

\author{
Spartak Spasov Yanakiev (iD) ${ }^{1}$ and Mirela Borislavova Marinova-Takorova $\mathbb{D}^{2}$ \\ ${ }^{1}$ Medical College, Medical University of Sofia, Yordanka Filaretova Str. No. 3, Sofia, Bulgaria \\ ${ }^{2}$ Department of Conservative Dentistry, Faculty of Dental Medicine, Medical University of Sofia, Georgi Sofiiski 3 Str., Sofia, Bulgaria
}

Correspondence should be addressed to Spartak Spasov Yanakiev; s.yanakiev@mc.mu-sofia.bg

Received 16 March 2021; Accepted 12 June 2021; Published 23 June 2021

Academic Editor: Konstantinos Michalakis

Copyright (C) 2021 Spartak Spasov Yanakiev and Mirela Borislavova Marinova-Takorova. This is an open access article distributed under the Creative Commons Attribution License, which permits unrestricted use, distribution, and reproduction in any medium, provided the original work is properly cited.

\begin{abstract}
A four-year follow-up of a novel silane heat treatment method for bonding lithium disilicate overlays to tooth structures without hydrofluoric acid etching of the ceramic surface is presented in this case report. Silane heat treatment modifies the silane layer and thus enhances resin ceramic bond strength without hydrofluoric acid etching. The standard ceramic preparation technique prior to bonding silicate ceramics to tooth structure is hydrofluoric acid etching and applying a silane coupling agent, followed by dental adhesive. In this case, the micromechanical roughening of the ceramic surface was performed by air abrasion with $\mathrm{Al}_{2} \mathrm{O}_{3}$. Silane heat treatment with constant $120^{\circ} \mathrm{C}$ airflow, applied for $60 \mathrm{sec}$, followed by dental adhesive application enhanced the resinceramic bond strength. After a four-year follow-up, the restorations' clinical appearance could be defined as excellent/very good according to the FDI clinical criteria for the evaluation of direct and indirect restorations. This clinical result supports many in vitro studies regarding the resin-ceramic bond strength and durability obtained through postsilanization heat treatment.
\end{abstract}

\section{Introduction}

Lithium disilicate $\left(\mathrm{LiS}_{2}\right)$ is one of the most widely applied metal-free materials due to its high aesthetics, adequate mechanical properties, and favorable bonding strength, ensuring functional durability and high short- and medium-term survival rates [1-4]. It can be used for both tooth- and implant-supported restorations, including single crowns, bridges, anterior veneers, posterior inlays, onlays, and overlays [5]. In cases when single restorations like inlays, onlays, and partial crowns are needed, they present advantages such as high fracture resistance, low wear and abrasive potential, biocompatibility, adhesive bonding, and strength which make them preferable in cases with significant loss of tooth structure and abrasion or when occlusal correction is needed [5]. Bonded restorations with full occlusal coverage preserve tooth structure compared to full-coverage crowns and increase the fracture resistance of endodontically treated teeth compared to direct MOD restorations [6]. $\mathrm{LiS}_{2}$ has shown promising clinical results for posterior endodontically treated teeth with or without fiber posts [7]. These restorations' success depends on many factors-tooth preparation, presence of enamel, bonding procedures, and material selection $[8,9]$.

The adequate adhesive bonding procedure for glassceramics needs proper preparation of the inner surface of the restoration. The significant adhesive strength is due to both micromechanical and chemical bonding mechanisms. The surface microirregularities for the micromechanical interlocking between the ceramic and the cement could be made by acid etching, sandblasting, or diamond bur grinding [5]. The application of a silane coupling agent ensures chemical interaction between the ceramics and the adhesive resin [10].

Hydrofluoric acid (HF) etching of glass-ceramics proved very efficient since it creates a large bonding surface $[11,12]$. On the other hand, if it comes in contact with skin, eyes, or mucosa, HF could be harmful to both the patient and the clinician $[13,14]$ and is acknowledged as a highly hazardous chemical in dentistry $[15,16]$. That is why some authors exert efforts on improving the chemical bond between dental ceramics and composite resin provided by the silane coupling 


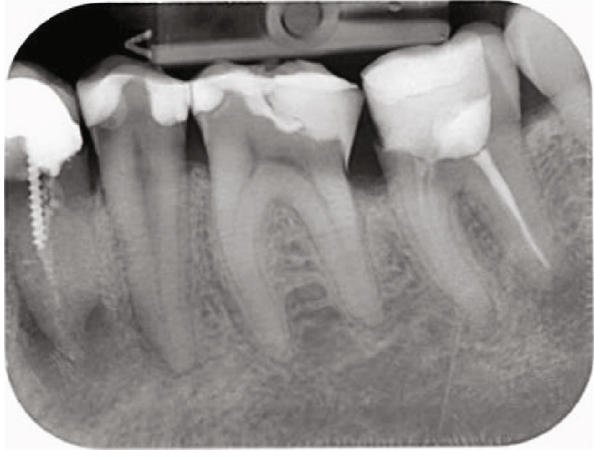

(a)

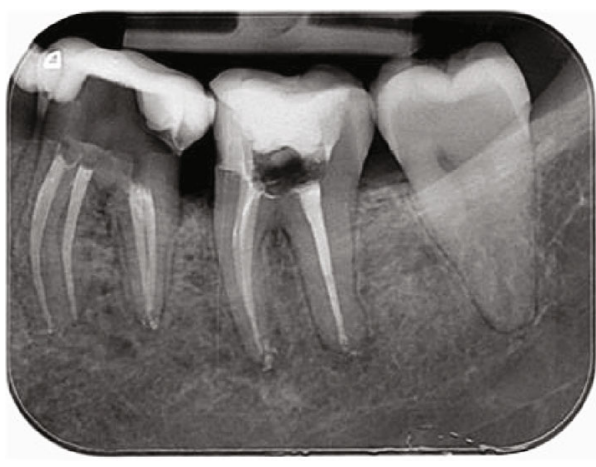

(c)

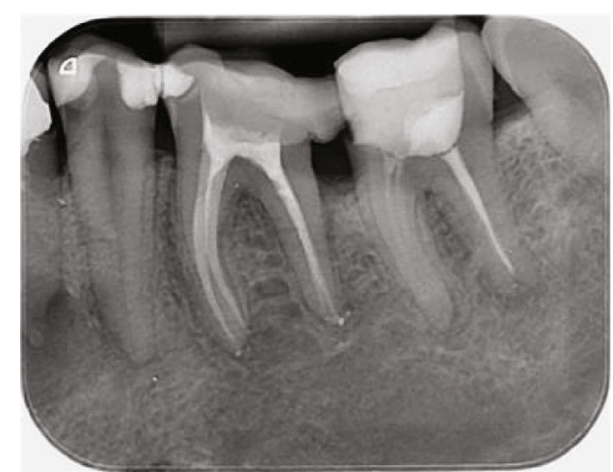

(b)

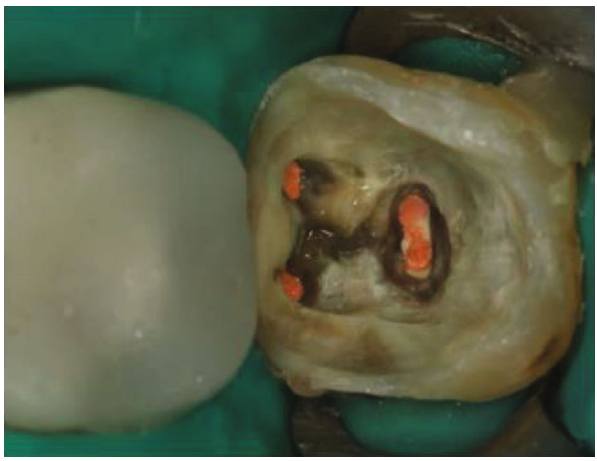

(d)

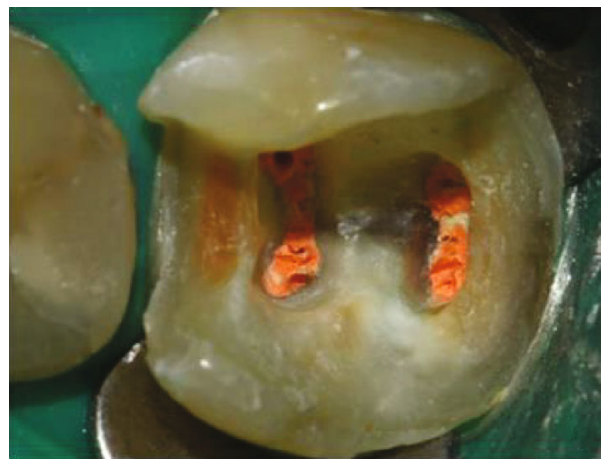

(e)

FIGURE 1: Preoperative images: radiograph images of tooth 36 (a) and 37 (b) prior to endodontic treatment and postendodontic treatment (c) and clinical view of tooth 37 (d) and 36 (e).

agents, thus trying to eliminate the use of HF acid. Silane treatment with heated airflow or in an oven is one of the most frequently used methods [17-20]. The proposed heating temperatures vary from 38 to $100^{\circ} \mathrm{C}$. In a recent study, a $120^{\circ} \mathrm{C}$ heating temperature of the silane was presented [21]. This temperature was the most efficient treatment method, providing the highest bond strength among all heating temperatures. According to some studies, HF's effect in the bonding procedure could be compensated by micromechanical roughening of the ceramic restoration and silane heat treatment to obtain high bond strength $[17,18]$. Despite the large number of in vitro studies in the literature, there is still a lack of clinical evidence of these methods' effectiveness in vivo.

This clinical case report describes two ceramic overlays' bonding procedures made from $\mathrm{LiS}_{2}$ without $\mathrm{HF}$ etching and a 4-year follow-up.

\section{Case Description}

The patient is a 34-year-old male, a smoker with good oral hygiene, who presented into our office for a routine examination. There was secondary caries around a composite resin restoration on tooth 36 . The $\mathrm{X}$-ray image of the tooth verified secondary caries on tooth 36 and revealed poor marginal adaptation of the restoration and an inadequate root canal treatment of tooth 37. After discussing the clinical options, the patient signed informed consent-root canal treatment and ceramic overlay restorations of both teeth were planned.

The endodontic treatment of the teeth was performed under microscope Zumax OMS2350 (Zumax Medical Co., Ltd., Suzhou New District, China) in several visits one by one. Root canals were shaped with MTwo files, size 25/0.06 (VDW GmbH, Munich, Germany). After copious irrigation 


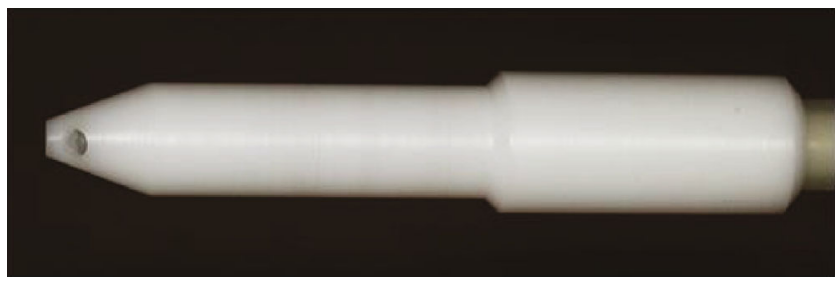

(a)

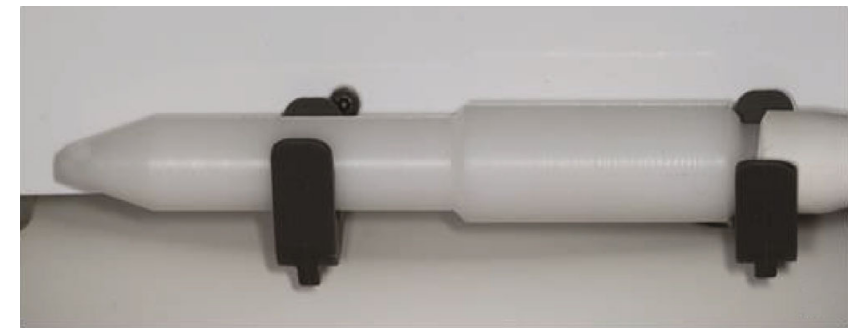

(b)

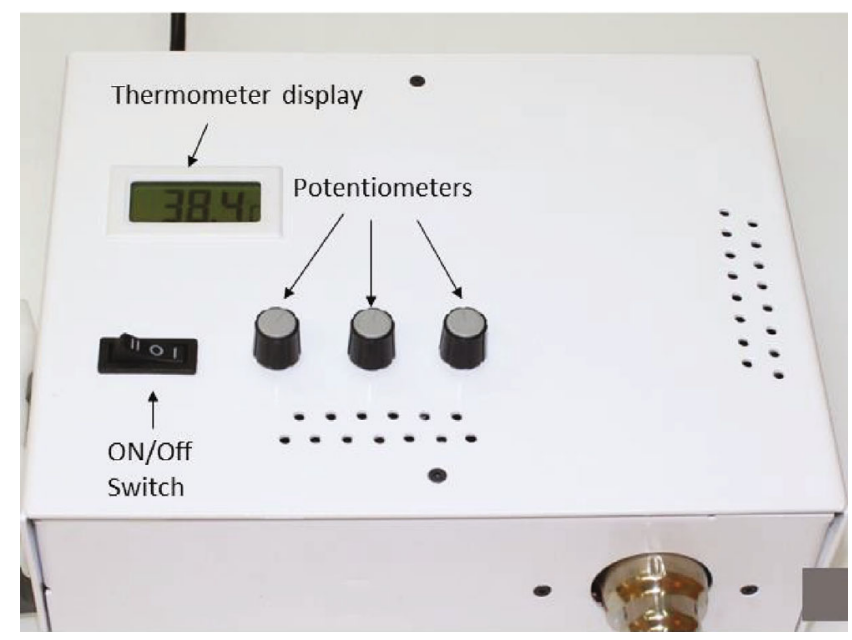

(c)

Figure 2: The custom-made air machine used in this study. Autoclavable handpiece made from polyacetate material (placed on the side wall of the device) ( $\mathrm{a}, \mathrm{b})$. Front panel of the device and basic components (c).

with $\mathrm{NaOCl}$ solution (Chloraxid 2\%, Cerkamed, Stalowa Wola, Poland), the final irrigation with EDTA solution (MD-Cleanser, Meta Biomed Europe $\mathrm{GmbH}$, Mülheim an der Ruhr, Germany) and ethanol 95\% (Chemax Pharma, Sofia, Bulgaria) was done before filling the canals with $\mathrm{AH}$ plus root canal sealer (Dentsply DeTrey, GmbH, Konstanz, Germany) and taper-matched gutta-percha cone (Sure Dent, Sagimakgol-ro, Jungwon-gu, Seongnam-si, Gyeonggi-do, South Korea) utilizing a single-cone technique (Figure 1).

Tooth preparation for the overlays was performed, and provisional acrylic restorations were placed (Unifast III, GC Corporation, Tokyo, Japan). Full-tray maxillary and mandibular impressions were taken on the next visit using VPS material (Variotime, Heraeus Kulzer GmbH, Hanau, Germany). Two $\mathrm{LiS}_{2}$ overlays were fabricated from E.max Press, shade A 3.5 HT (Ivoclar Vivadent, Schaan, Liechtenstein).

The two ceramic overlays were air abraded with $50 \mu \mathrm{m}$ $\mathrm{Al}_{2} \mathrm{O}_{3}$ at 1 bar. After the try-in procedures, the inner surfaces of the overlays were cleaned with ethanol and air-dried. A silane coupling agent (Monobond Plus, Ivoclar Vivadent, Schaan, Liechtenstein) was applied for 60 seconds. The key step in the protocol was the silane heat treatment performed with a custom-made hot air machine (Figure 2). The device is equipped with an autoclavable handpiece made from polyacetate material TECAFORM AH POM (Modern Plastics, Connecticut, USA). A thermocouple located in the working end of the handpiece measures the air temperature as near as possible to the treated surface. Temperature is measured to the first decimal place in degrees Celsius. The temperature range supported by the device is from $38^{\circ} \mathrm{C}$ to $140^{\circ} \mathrm{C}$.

A constant $120^{\circ} \mathrm{C}$ airflow for $60 \mathrm{sec}$ was applied, followed by dental adhesive OptiBond FL (Kerr Italia S.r.l., Scafati (SA), Italy). The steps are presented in Figure 3.

The tooth structures were etched with $37.5 \%$ phosphoric acid (LOT 6775164, Gel Etchant, Kerr Italia S.r.l., Scafati (SA), Italy), and an OptiBond FL dental adhesive was applied. A flowable composite (Gaeneal Universal Flow, GC Corporation, Tokyo, Japan) was used for the permanent fixation. Light curing for $60 \mathrm{sec}$ on each tooth surface was performed with Elipar ${ }^{\mathrm{TM}}$ DeepCure-S LED Curing Light (3M ESPE, St. Paul, USA). Occlusal contact check and adjustments were done after the final polymerization with $40 \mu \mathrm{m}$ articulating paper (Bausch Arti-Check, Dr. Jean Bausch $\mathrm{GmbH} \&$ Co. KG, Koln, Germany). The final result is shown in Figure 4.

The FDI clinical criteria for the evaluation of direct and indirect restorations were used to evaluate the clinical appearance of the restorations after four years in use [22]. The appearance of the restorations could be assessed as clinically excellent/very good based on the aesthetic, functional, and biological properties. Lustre is comparable to that of enamel, and no surface staining, good color match, and ideal form preservation were observed. The absence of fractures, excellent marginal adaptation, normal contact points, and patient satisfaction correspond to clinical excellent/very good functional properties. The radiograph examination reveals a 


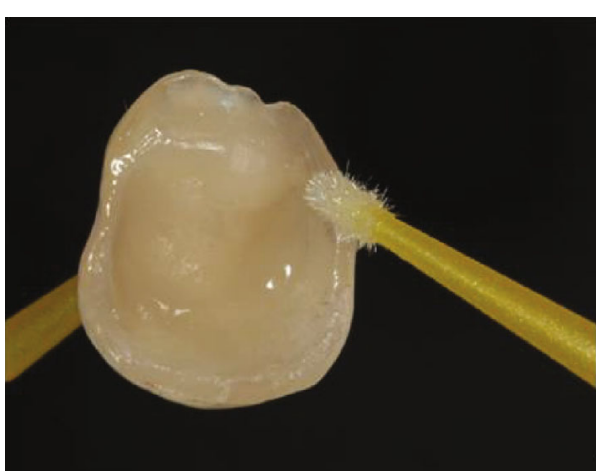

(a)

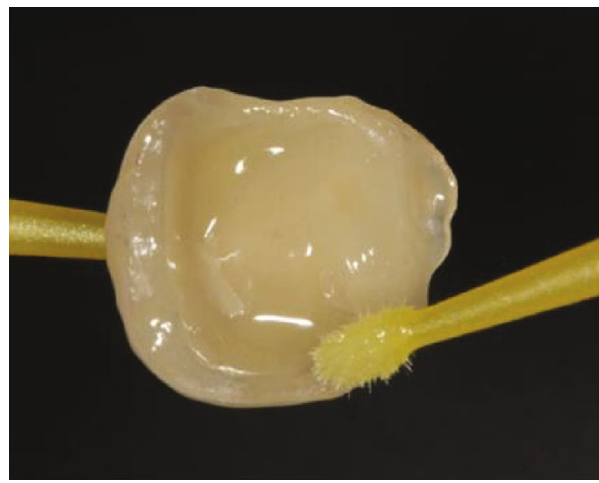

(c)

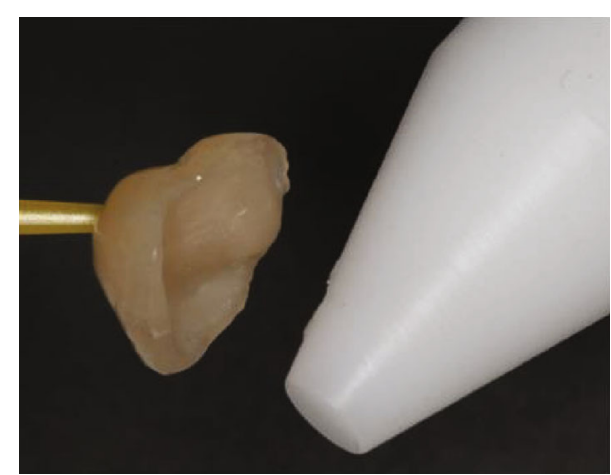

(b)

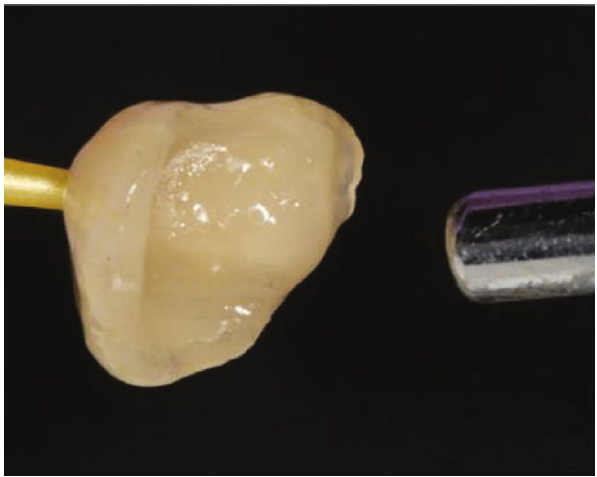

(d)

Figure 3: Bonding procedure: silane application (a); postsilanization heat treatment with custom-made device (b); bonding agent application (c); bonding agent solvent evaporation (d).

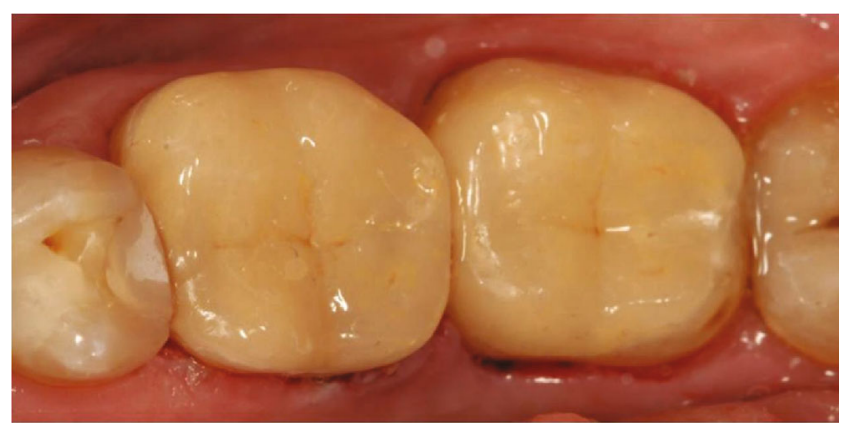

FIGURE 4: Postoperative view of the two overlays immediately after fixation and isolation removal.

harmonious transition between the restorations and the tooth structures. We observed no hypersensitivity, no secondary or primary caries, very good periodontal response, healthy mucosa, and no general oral symptoms (Figure 5).

\section{Discussion}

This clinical result supports many in vitro studies regarding the resin-ceramic bond strength and durability obtained through postsilanization heat treatment.

The adhesive bonding procedure is one of the most critical steps determining the success and durability of $\mathrm{LiS}_{2}$ restorations. Proper tooth and ceramic preparation is mandatory. HF etching of the ceramic surface, followed by silane and dental adhesive application, is usually recommended for establishing a strong resin-ceramic bond. The HF etching dissolves partially the glassy phase of the silica-based ceramics, providing micromechanical retentions [23]. More undercuts and larger surface characterize etched ceramic compared to air-abraded ceramic; thus, a stronger resinceramic bond could be obtained [11, 24].

$\mathrm{HF}$ is a strong ceramic etching agent but potentially very harmful if it comes in contact with eyes, skin, or mouth tissues [16]. For this reason, some authors propose other etching agents, which are less effective, or to avoid the use of $\mathrm{HF}$ in the bonding procedure and use alternative protocols [25]. Despite the advantages of HF etching, it could weaken the material if the clinician exceeds the recommended etching time [26]. Overetching could also weaken the ceramic-composite bond strength [27]. HF promotes selective dissolution of the glassy phase of silicabased ceramics. The etching process includes several chemical reactions that lead to the deposition of insoluble silica-fluoride salts on the surface that negatively affects the bond. Thus, proper postetching cleaning of the ceramic is mandatory [28]. One could avoid these possible complications of the bonding procedure by excluding the HF etching in the bonding protocol. If so, to enhance the bond strength, proper postsilanization heat treatment combined with an appropriate microbraided ceramic surface is mandatory. To obtain a more durable resin-ceramic bond in the presented case, we applied postsilanization heat treatment at $120^{\circ} \mathrm{C}$ and avoided $\mathrm{HF}$ etching. 


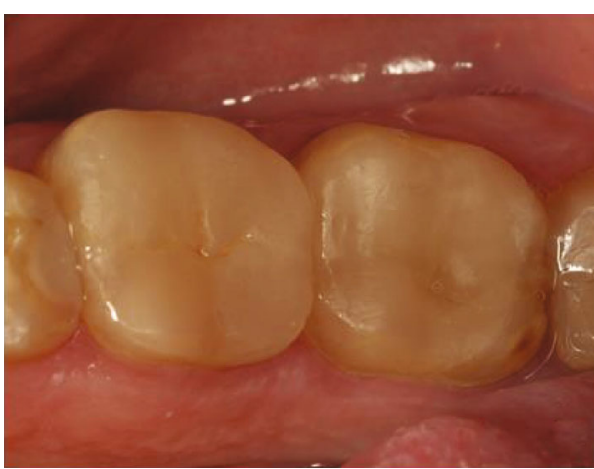

(a)

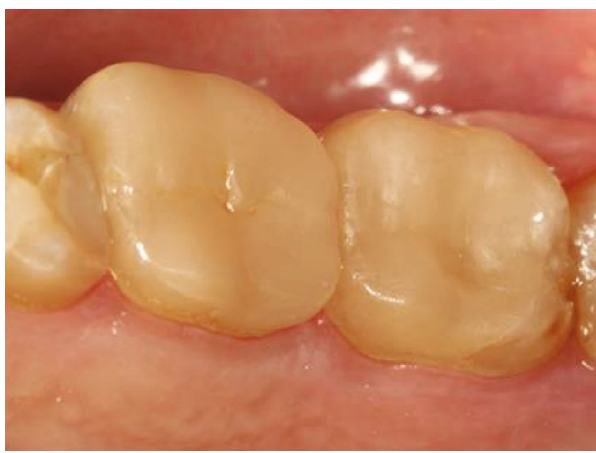

(c)

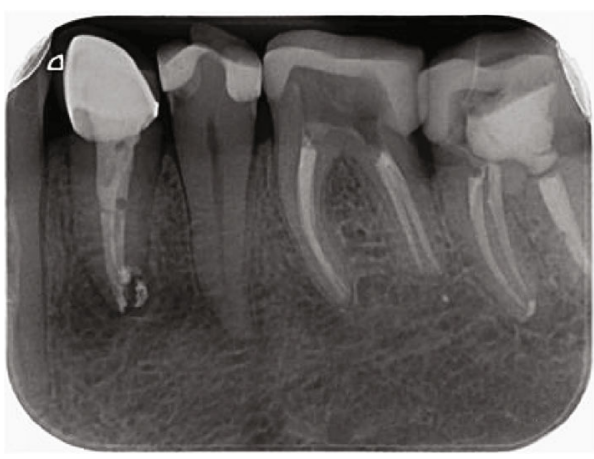

(e)

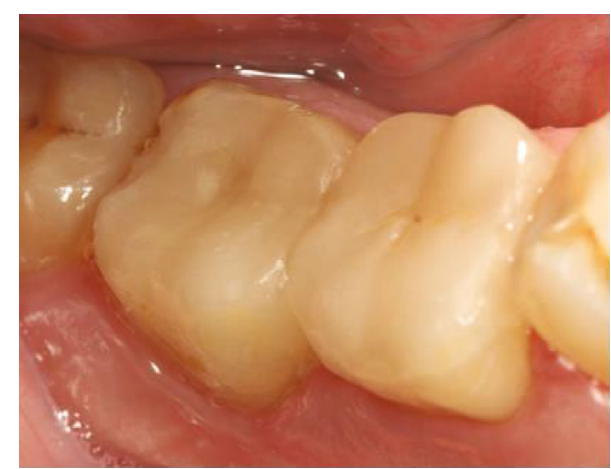

(b)

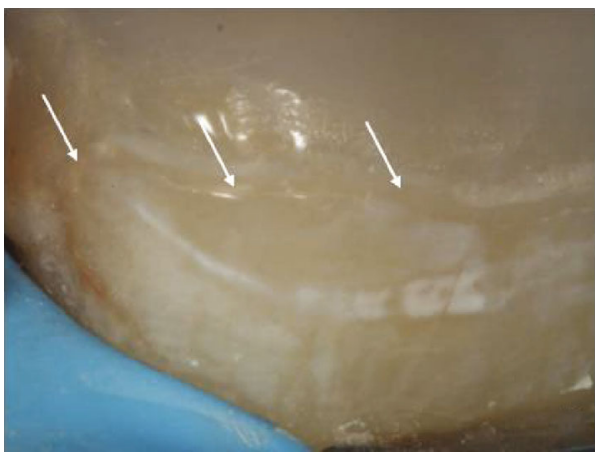

(d)

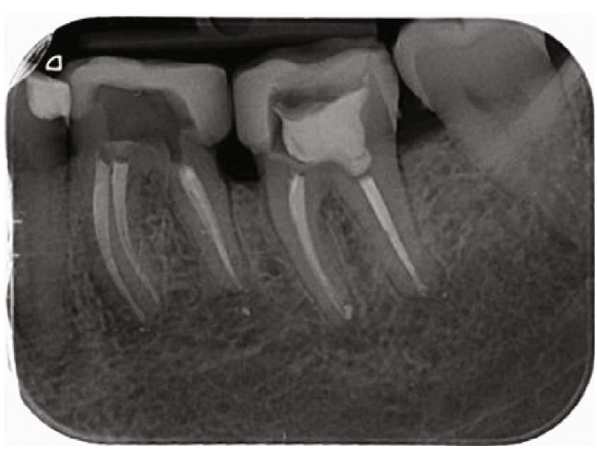

(f)

FiguRE 5: Four-year follow-up of the restorations: occlusal view (a); buccal view (b); lingual view (c); a close-up image of the overlay-tooth interface (d), white arrows pointing to the borders of the restorations; radiograph examination (e, $f)$.

In addition to the micromechanical retentions, silane molecules can bond to both the functional groups of the silica phase of the ceramic and the resin material's organic matrix, thus providing chemical bonding between the two materials [29]. The efficacy of the chemical bonding depends on the type of the silane coupling agent, the material's application, and postsilanization treatment [30]. An interlayer is formed when a silane coupling agent is applied on the ceramic surface before resin application. Three different structures could be identified in the silane interlayer. The outermost layer comprises small oligomers, which could be easily eliminated by organic solvents and water at room temperature. The middle layer is formed by oligomers connected with stronger single siloxane bonds. In the inner layer, silane molecules form a network characterized by higher hydrolytic stability. Silane layer thickness should be as minimal as possible, and only the inner molecule layer is needed to obtain a strong and durable resin-ceramic bond. One of the most effective ways to obtain a highly condensed and stronger silane layer is postsilanization heat treatment [31].

Recommended temperatures vary among different authors. Fabianeli et al. and Roulet et al. propose $100^{\circ} \mathrm{C}$ heating temperature $[17,32]$. Monticelli et al. use $38^{\circ} \mathrm{C}$ [33]. Hooshmand et al. tested three different temperatures $-100^{\circ} \mathrm{C}$ (in oven), $80^{\circ} \mathrm{C}$ (boiled water), and $50^{\circ} \mathrm{C}$ (hot air) [34]. A recent study conducted a systematic comparison between different postsilanization heating temperatures. It showed the positive effect of higher temperatures on the bond strength between feldspathic ceramics and resin composite material [21]. The highest tensile bond strength was achieved among all groups when the bonding surface was treated with $120^{\circ} \mathrm{C}$ hot air. Since this was the most effective silane heat 
treatment method, we chose it appropriately in this clinical case. The $60 \mathrm{sec}$ application of this temperature ensures a high condensation rate of the silane molecules; thus, a stronger chemical bond is achieved. It is harmless for both the patient and the clinician since the heating process is performed outside the mouth and the overlays are attached on a bonding stick.

Despite the very good outcome of the presented bonding method, there are some limitations. The follow-up period is relatively short (4 years). Different follow-up periods are reported in the literature-usually from 12 months to 15 years [2-4]. Thus, although the reported results are quite satisfactory, they should be considered preliminary. Since this case report presents only one patient and two overlays, it could not be accepted as scientific proof. Therefore, it could only be assumed as a promising result, and further randomized control trials should validate this approach and confirm the reliability of this method in vivo. Another limitation is the need for a specially designed hot air device, which is not supported by any manufacturer as far as we know.

\section{Conclusion}

The clinical appearance of the restorations could be defined as excellent/very good according to the FDI clinical criteria for the evaluation of direct and indirect restorations for the four-year follow-up period. Thus, it could be assumed that the bonding protocol used in this clinical case promotes a strong and durable ceramic-composite bond due to the combination of micromechanical retentions provided by air abrasion with $\mathrm{AL}_{2} \mathrm{O}_{3}$ and proper silane heat treatment. Although the results are promising, further studies should be conducted due to the limitations of this case report.

\section{Conflicts of Interest}

The authors declare no conflict of interest.

\section{References}

[1] M. Luciano, Z. Francesca, S. Michela, M. Tommaso, and A. Massimo, "Lithium disilicate posterior overlays: clinical and biomechanical features," Clinical Oral Investigations, vol. 24, no. 2, pp. 841-848, 2020.

[2] A. Moreira, F. Freitas, D. Marques, and J. Caramês, "Aesthetic rehabilitation of a patient with bruxism using ceramic veneers and overlays combined with four-point monolithic zirconia crowns for occlusal stabilization: a 4-year follow-up," Case Reports in Dentistry, vol. 2019, Article ID 1640563, 7 pages, 2019.

[3] C. van den Breemer, C. Vinkenborg, H. van Pelt, D. Edelhoff, and M. S. Cune, "The clinical performance of monolithic lithium disilicate posterior restorations after 5,10 , and 15 years: a retrospective case series," The International Journal of Prosthodontics, vol. 30, no. 1, pp. 62-65, 2017.

[4] G. Fabbri, F. Zarone, G. Dellificorelli et al., "Clinical evaluation of 860 anterior and posterior lithium disilicate restorations: retrospective study with a mean follow-up of 3 years and a maximum observational period of 6 years," The International
Journal of Periodontics \& Restorative Dentistry, vol. 34, no. 2, pp. 165-177, 2014.

[5] F. Zarone, M. I. di Mauro, P. Ausiello, G. Ruggiero, and R. Sorrentino, "Current status on lithium disilicate and zirconia: a narrative review," BMC Oral Health, vol. 19, no. 1, p. 134, 2019.

[6] G. T. Rocca and I. Krejci, "Crown and post-free adhesive restorations for endodontically treated posterior teeth: from direct composite to endocrowns," The European Journal of Esthetic Dentistry, vol. 8, no. 2, pp. 156-179, 2013.

[7] M. Ferrari, E. Ferrari Cagidiaco, C. Goracci et al., "Posterior partial crowns out of lithium disilicate (LS2) with or without posts: a randomized controlled prospective clinical trial with a 3-year follow up," Journal of Dentistry, vol. 83, pp. 12-17, 2019.

[8] G. Politano, B. Van Meerbeek, and M. Peumans, "Nonretentive bonded ceramic partial crowns: concept and simplified protocol for long-lasting dental restorations," The Journal of Adhesive Dentistry, vol. 20, no. 6, pp. 495-510, 2018.

[9] G. T. Rocca, R. Daher, C. M. Saratti et al., "Restoration of severely damaged endodontically treated premolars: the influence of the endo-core length on marginal integrity and fatigue resistance of lithium disilicate CAD-CAM ceramic endocrowns," Journal of Dentistry, vol. 68, pp. 41-50, 2018.

[10] R. Frankenberger, V. E. Hartmann, M. Krech et al., "Adhesive luting of new CAD/CAM materials," International Journal of Computerized Dentistry, vol. 18, no. 1, pp. 9-20, 2015.

[11] A. Della Bona and K. J. Anusavice, "Microstructure, composition, and etching topography of dental ceramics," The International Journal of Prosthodontics, vol. 15, no. 2, pp. 159-167, 2002.

[12] N. Mobilio, A. Fasiol, and S. Catapano, "Qualitative evaluation of the adhesive interface between lithium disilicate, luting composite and natural tooth," Ann Stomatol (Roma), vol. 7, no. 1-2, pp. 1-3, 2016.

[13] W. J. Anderson and J. R. Anderson, "Hydrofluoric acid burns of the hand: mechanism of injury and treatment," The Journal of Hand Surgery, vol. 13, no. 1, pp. 52-57, 1988.

[14] F. Caliskan Tur and E. Aksay, "Hydrofluoric acid exposure," Turk J Emerg Med, vol. 15, no. 1, p. 1, 2015.

[15] T. L. Litovitz, W. Klein-Schwartz, K. S. Dyer, M. Shannon, S. Lee, and M. Powers, "1997 annual report of the American Association of Poison Control Centers Toxic Exposure Surveillance System," The American Journal of Emergency Medicine, vol. 16, no. 5, pp. 443-497, 1998.

[16] M. Ozcan, A. Allahbeickaraghi, and M. Dündar, "Possible hazardous effects of hydrofluoric acid and recommendations for treatment approach: a review," Clinical Oral Investigations, vol. 16, no. 1, pp. 15-23, 2012.

[17] A. Fabianelli, S. Pollington, F. Papacchini et al., "The effect of different surface treatments on bond strength between leucite reinforced feldspathic ceramic and composite resin," Journal of Dentistry, vol. 38, no. 1, pp. 39-43, 2010.

[18] C. Shen, W. S. Oh, and J. R. Williams, "Effect of postsilanization drying on the bond strength of composite to ceramic," The Journal of Prosthetic Dentistry, vol. 91, no. 5, pp. 453-458, 2004.

[19] C. Cotes, R. F. de Carvalho, E. T. Kimpara, F. P. Leite, and M. Ozcan, "Can heat treatment procedures of prehydrolyzed silane replace hydrofluoric acid in the adhesion of resin cement to feldspathic ceramic?," The Journal of Adhesive Dentistry, vol. 15, no. 6, pp. 569-574, 2013. 
[20] R. F. de Carvalho, C. Cotes, E. T. Kimpara, F. P. Leite, and M. Özcan, "Heat treatment of pre-hydrolyzed silane increases adhesion of phosphate monomer-based resin cement to glass ceramic," Brazilian Dental Journal, vol. 26, no. 1, pp. 44-49, 2015.

[21] Medical College"Y Filaretova", Medical University - Sofia, Bulgaria, S. Yanakiev, B. Yordanov, and V. Dikov, "Influence of silane heat treatment on the tensile bond strength between EX-3 synthetic veneering porcelain and composite resin using five different activation temperatures," Journal of IMAB Annual Proceeding (Scientific Papers), vol. 23, no. 1, pp. 1456-1459, 2017.

[22] R. Hickel, A. Peschke, M. Tyas et al., "FDI World Dental Federation: clinical criteria for the evaluation of direct and indirect restorations-update and clinical examples," Clinical Oral Investigations, vol. 14, no. 4, pp. 349-366, 2010.

[23] M. C. Bottino, M. Özcan, P. G. Coelho, L. F. Valandro, J. C. Bressiani, and A. H. Bressiani, "Micro-morphological changes prior to adhesive bonding: high-alumina and glassy-matrix ceramics," Brazilian Oral Research, vol. 22, no. 2, pp. 158-163, 2008.

[24] S. Canay, N. Hersek, and A. Ertan, "Effect of different acid treatments on a porcelain surface," Journal of Oral Rehabilitation, vol. 281, pp. 95-101, 2001.

[25] M. Ozcan, "Evaluation of alternative intra-oral repair techniques for fractured ceramic-fused-to-metal restorations," Journal of Oral Rehabilitation, vol. 30, no. 2, pp. 194-203, 2003.

[26] T. Hooshmand, S. Parvizi, and A. Keshvad, "Effect of surface acid etching on the biaxial flexural strength of two hotpressed glass ceramics," Journal of Prosthodontics, vol. 17, no. 5, pp. 415-419, 2008.

[27] J. H. Chen, H. Matsumura, and M. Atsuta, "Effect of etchant, etching period, and silane priming on bond strength to porcelain of composite resin," Operative Dentistry, vol. 23, no. 5, pp. 250-257, 1998.

[28] G. Bruzi, A. O. Carvalho, M. Giannini, H. P. Maia, and P. Magne, "Post-etching cleaning influences the resin shear bond strength to CAD/CAM lithium-disilicate ceramics," Appl Adhes Sci, vol. 5, no. 1, 2017.

[29] R. Li, S. Q. Ma, C. C. Zang et al., "Enhanced bonding strength between lithium disilicate ceramics and resin cement by multiple surface treatments after thermal cycling," PLoS One, vol. 14, no. 7, article e0220466, 2019.

[30] J. P. Matinlinna, L. V. Lassila, M. Ozcan, A. Yli-Urpo, and P. K. Vallittu, "An introduction to silanes and their clinical applications in dentistry," The International Journal of Prosthodontics, vol. 17, no. 2, pp. 155-164, 2004.

[31] E. Metwalli, D. Haines, O. Becker, S. Conzone, and C. G. Pantano, "Surface characterizations of mono-, di-, and triaminosilane treated glass substrates," Journal of Colloid and Interface Science, vol. 298, no. 2, pp. 825-831, 2006.

[32] J. F. Roulet, K. J. M. Söderholm, and J. Longmate, "Effects of treatment and storage conditions on ceramic/composite bond strength," Journal of Dental Research, vol. 74, no. 1, pp. 381387, 1995.

[33] F. Monticelli, M. Toledano, R. Osorio, and M. Ferrari, "Effect of temperature on the silane coupling agents when bonding core resin to quartz fiber posts," Dental Materials, vol. 22, no. 11, pp. 1024-1028, 2006.

[34] T. Hooshmand, R. van Noort, and A. Keshvad, "Bond durability of the resin-bonded and silane treated ceramic surface," Dental Materials, vol. 18, no. 2, pp. 179-188, 2002. 